\title{
Anomalías Vasculares de la Cavidad Oral: Revisión de la Clasificación y Tratamiento Aplicado a dos Casos Clínicos
}

\author{
Vascular Anomalies of the Oral Cavity: Review of Classification \\ and Treatment Applied to Two Clinical Cases
}

Coral Torres M. ${ }^{1}$; Josefina Santana L. ${ }^{1}$; Rodrigo Bravo A. ${ }^{1,2,3}$ \& Marcelo Mardones M. ${ }^{1,2,3}$

TORRES, M. C.; SANTANA, L. J.; BRAVO, A. R. \& MARDONES, M. M. Anomalías vasculares de la cavidad oral: Revisión de la clasificación y tratamiento aplicado a dos casos clínicos. Int. J. Odontostomat., 14(1):48-54, 2020.

RESUMEN: Las anomalías vasculares de cabeza y cuello son un grupo de lesiones que afectan vasos sanguíneos y linfáticos donde el tratamiento sigue siendo un desafío. La clasificación actualizada de anomalías vasculares de cabeza y cuello es la clasificación de Mulliken modificada, que las subdivide en a) tumores vasculares y, b) malformaciones vasculares. En este reporte, presentamos dos casos clínicos de pacientes de sexo masculino, con diagnóstico de anomalías vasculares que afectan al labio y paladar duro, diagnosticados como malformación arteriovenosa y malformación venosa, respectivamente. Dichas lesiones remitieron completamente mediante tratamientos conservadores (agentes esclerosantes) y/o quirúrgicos (exéresis quirúrgica completa de la lesión) logrando una remisión completa. Consecutivamente, presentamos una revisión de la literatura enfocado a la clasificación actual, enfoques terapéuticos actuales y futuros.

PALABRAS CLAVE: anomalías vasculares, malformaciones vasculares, tumores vasculares, cavidad oral.

\section{INTRODUCCIÓN}

Las anomalías vasculares (AV) son un grupo de lesiones derivadas de vasos sanguíneos y linfáticos que incluyen tanto a los tumores vasculares como a las malformaciones vasculares. Históricamente, se han realizado varios intentos para llegar a una clasificación definitiva de estas condiciones. Inicialmente en 1982, Mulliken y Glowacki describieron una clasificación biológica de las AV, basada tanto en las características patológicas predominantes del endotelio como en su evolución natural (Redondo, 2004). En abril del año 2014, se presentó un sistema de clasificación más reciente y completo para estas anomalías, sub-dividiéndolas en tumores vasculares y malformaciones vasculares (Gupta et al., 2017). Sin embargo la clasificación de Mulliken modificada es la más aceptada hoy en día para las anomalías vasculares de cabeza y cuello (Elias et al., 2016), debido a que proporciona una clasificación simple pero completa de las lesiones vasculares de la infancia y la niñez, y sirve como una guía para el diagnóstico, el tratamiento y la investigación adicional (Mulliken \& Glowacki, 1982).
Los tumores vasculares comprenden los hemangiomas infantiles, hemangiomas congénitos y granuloma piógeno. Por otro lado, las malformaciones vasculares se subclasifican en malformaciones capilares, venosas, linfáticas y arteriovenosas.

El tratamiento de las AV sigue siendo un desafío, ya que dependen de un diagnóstico preciso y la participación de un equipo multidisciplinario (Kim et al., 2017). Dentro de los tratamientos se destacan tratamientos quirúrgicos que están indicados en los casos que comprometen la estética o interfieren con la función de la región involucrada, y tratamientos no quirúrgicos como terapia con corticoides, láser, embolización, crioterapia y uso de agentes esclerosantes (Cabrera \& Redondo, 2004).

El objetivo del presente trabajo es presentar dos casos clínicos de AV localizados en la cavidad oral tratados mediante un abordaje mixto, primero conservador mediante la inyección de un agente esclerosante

\footnotetext{
${ }^{1}$ Equipo Cirugía Maxilofacial, Servicio de Cirugía, Hospital San José, Santiago, Chile.

${ }^{2}$ Departamento de Cirugía y Traumatología Bucal y Maxilofacial, Facultad Odontología, Universidad de Chile, Santiago, Chile.

${ }^{3}$ Departamento de Cirugía Maxilofacial Clínica Las Condes, Santiago, Chile.
} 
y luego, la remoción quirúrgica. Consecutivamente, revisamos la literatura disponible enfocándonos en las clasificaciones que orientan el diagnóstico y las ventajas/desventajas de terapias disponibles.

\section{REPORTE DE CASO}

Caso 1. Paciente sexo masculino, 23 años, consulta por un aumento de volumen en labio superior ubicado en el lado izquierdo. La lesión presentaba 4 años de evolución, con periodos de crecimiento rápido, indoloro, y sin compromiso sensitivo local. Al examen físico, se constata el aumento de volumen en el labio superior en el lado izquierdo, de color violáceo, de $2 \times 3 \times 2$ centímetros aproximadamente, rodeado por mucosa y piel de aspecto normal. A la palpación, se comprueba indoloro, con límites netos, de consistencia blanda y sin pulso ni frémito (Fig. 1). A la ecografía Doppler, se comprobó una lesión sólida con múltiples estructuras vasculares arteriales de baja resistencia, y de alta velocidad de flujo. Debido al tamaño de la lesión y su ubicación, se decidió realizar un tratamiento inicial de infiltración local de $2 \mathrm{~mL}$ de un agente esclerosante (Polidocanol $1 \%$ ) en $2 \mathrm{~mL}$. Se realizaron cuatro infiltraciones locales perilesionales en cuatro sesiones separadas una semana. El tratamiento redujo del tamaño de la lesión, pero no la remitió por completo, obteniéndose un tamaño de 1,5 × $3 \times 1,5$ centímetros. Así, se decidió realizar la exéresis quirúrgica in toto de la lesión (Fig. 2) y estudio histopatológico subsecuente (Fig. 3). Durante la cirugía se ligaron y cauterizaron los vasos sanguíneos de suministro. El resultado del análisis histopatológico fue una proliferación vascular arteriovenosa benigna, concordante con malformación vascular. Paciente acude a control una semana posterior a la cirugía, con resultado funcional y estético favorable y satisfactorio (Fig. 4).
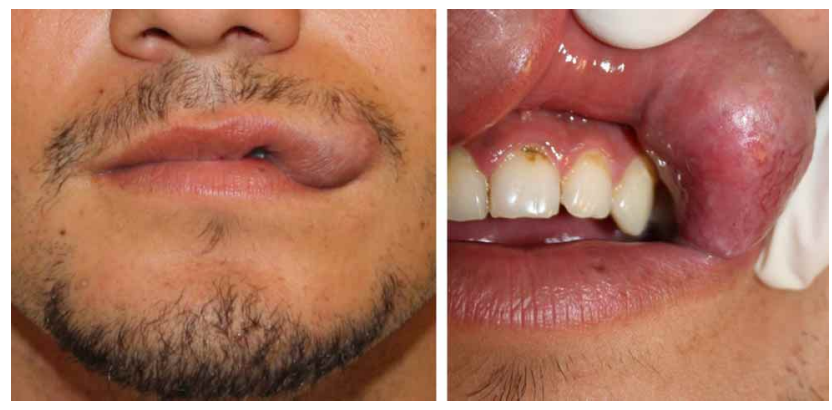

Fig.1. Aumento de volumen en labio superior lado izquierdo. Color violáceo, de $2 \times 3 \times 2 \mathrm{~cm}$ app, rodeado por mucosa y piel normal. A la palpación, indoloro, límites netos, consistencia blanda y sin pulso ni frémito.
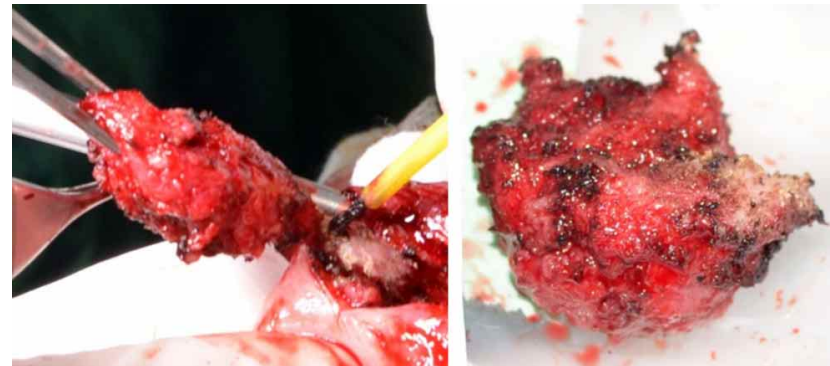

Fig. 2. Exéresis quirúrgica de la lesión.

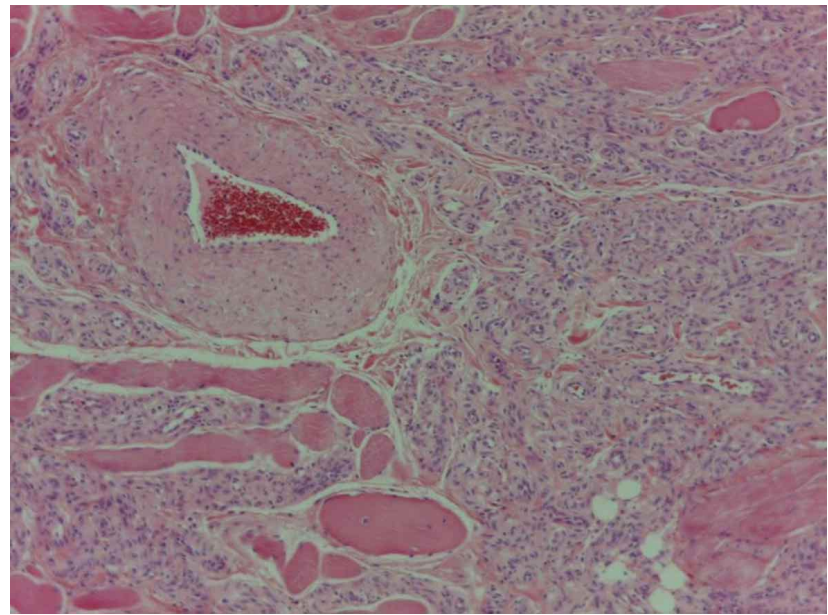

Fig. 3. Estudio histopatológico, tinción hematoxilina/eosina.

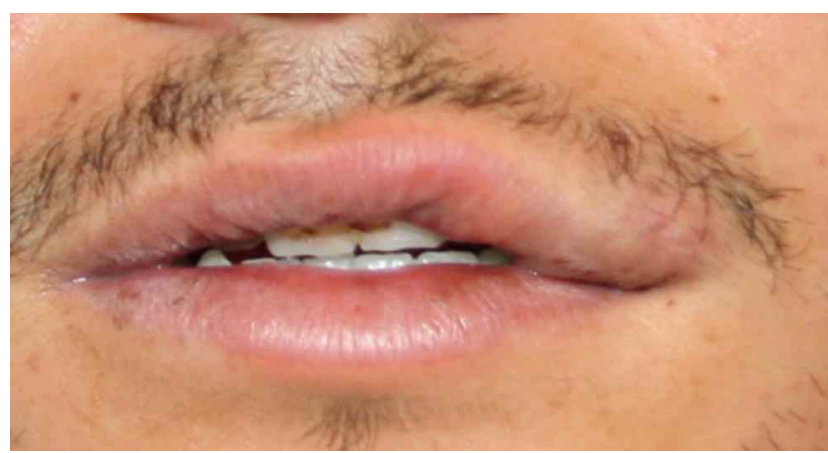

Fig. 4. Control post quirúrgico, 1 semana.

Caso 2. Paciente sexo masculino, 54 años, es derivado al servicio de Cirugía Maxilofacial del Hospital San José, Santiago, Chile, por lesión en paladar duro. Al examen clínico se observa lesión violácea, de 10 x 8 x $5 \mathrm{~mm}$, solevantada, márgenes definidos. A la palpación asintomática, ausencia de pulso y consistencia blanda (Fig. 5). Al realizar la maniobra de Valsalva, se observa expansión de la lesión. Las características clínicas y maniobras de diagnóstico sugieren diagnóstico de malformación venosa. La lesión es tratada de forma conservadora mediante infiltración intralesional de agente esclerosante de Polidocanol $2 \%$, en dos sesiones separadas por 1 semana. Controles poste- 
riores se evidencia disminución de tamaño de la malformación vascular y la mucosa palatina se presentó sin alteraciones (Fig. 6).

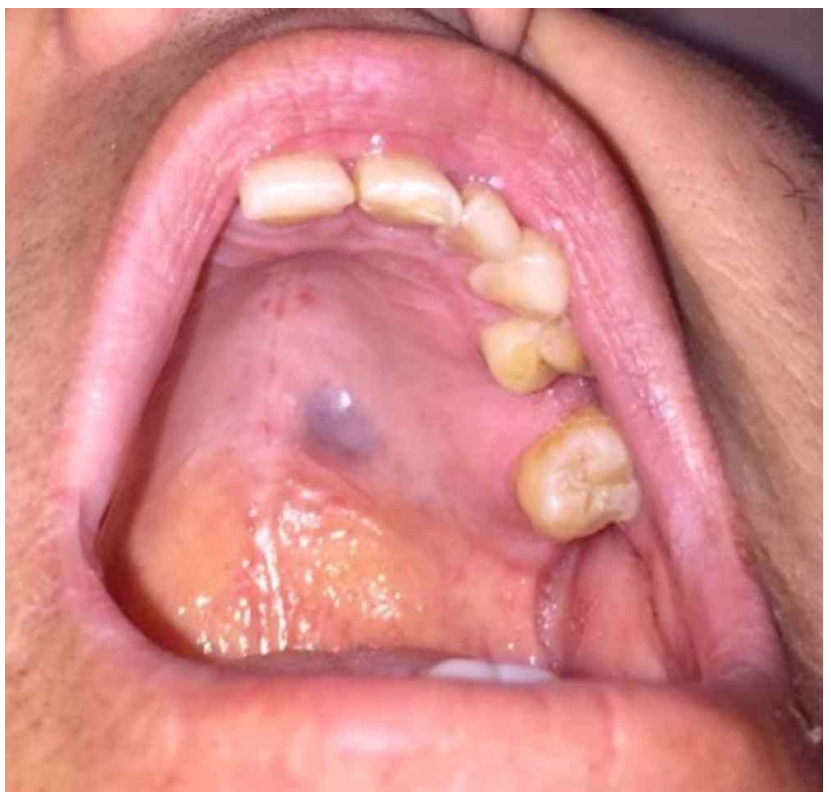

Fig. 5. Lesión en paladar duro, violácea, de 10 × 8 × 5 mm, solevantada, bordes definidos.

\section{DISCUSIÓN}

Las anomalías vasculares (AV) son un grupo de lesiones derivadas de vasos sanguíneos y linfáticos que presentan una histología variable y características de imagen / patología únicas, así como un comportamiento biológico distintivo (Medeiros et al., 2015). La clasificación más aceptada hoy en día es la "Clasificación de Mulliken modificada", que clasifica a las anomalías vasculares en tumores y malformaciones vasculares (Elias et al.).

Tumores vasculares. Son neoplasias verdaderas que exhiben proliferación de células patológicas endoteliales vasculares. Se subclasifican en: Hemangioma infantil, hemangioma congénito y granuloma piógeno.

Hemangioma infantil (HI). Tumores vasculares benignos generados por hiperplasia endotelial, no están presentes al nacer y aparecen en las primeras semanas de vida, tienen un rápido crecimiento en la infancia temprana seguido de una resolución lenta y espontánea dentro del tercer año de vida (Behnia et al., 2014). El 60 \% se localiza en cabeza y cuello, y aproximadamente 15 a $40 \%$ de los hemangiomas

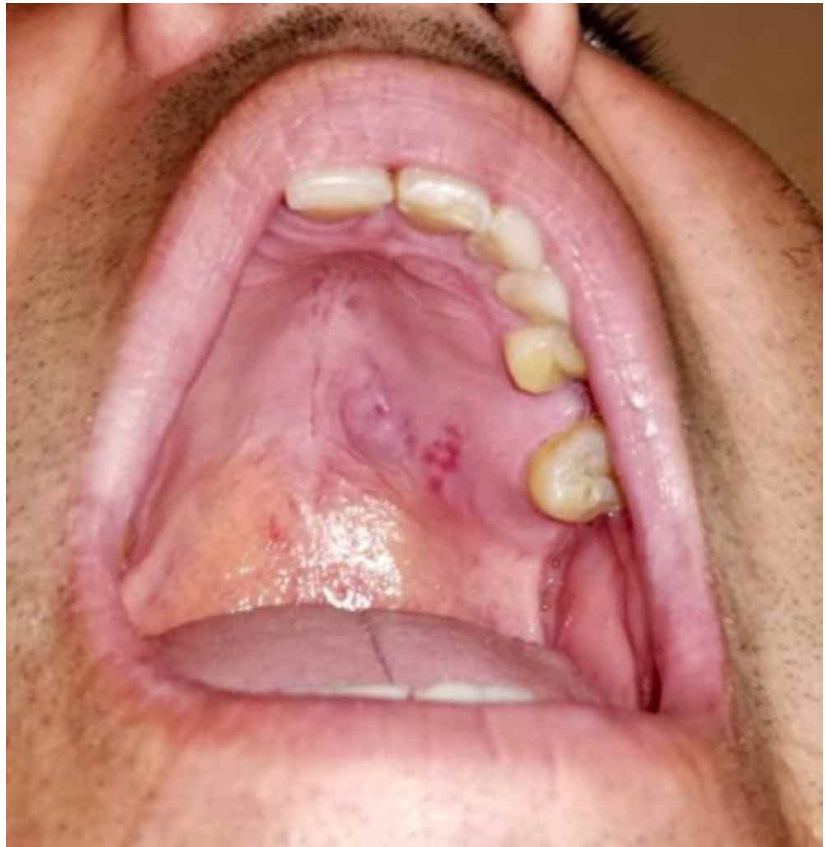

Fig. 6. Control posterior a tratamiento conservador mediante infiltración intralesional de agente esclerosante de Polidocanol $2 \%$. Se evidencia disminución de tamaño de la malformación vascular y mucosa palatina sin alteraciones.

involucionan espontáneamente, a menudo dejando cambios en la textura de la piel afectada (Frigerio \& Tan, 2015).

La mayoría de los HI, por su ubicación y tamaño, no requieren de tratamiento específico, dado su curso benigno y autolimitado. Sin embargo 10-15\% deben ser tratados en la fase proliferativa por riesgo a compromiso de estructuras vitales y alteraciones estéticas importantes (Léaute-Labrèze et al., 2008).

Hemangioma congénito (HC). Los hemangiomas congénitos se diferencian de los $\mathrm{HI}$ en que ya están maduros al nacer y no proliferan. Sólo representan el $3 \%$ de todos los hemangiomas (Elias et al.). Se subdividen en hemangiomas congénitos no involutivos ( $\mathrm{HCNI})$ y hemangiomas congénitos de rápida involución (HCRI) (Mulligan et al., 2014; da Silva et al., 2014).

Granuloma piógeno (GP). También conocido como hemangioma capilar lobular, es una lesión hiperplásica benigna de rápido crecimiento, de base sésil o pedunculada (Frigerio \& Tan). Se genera en respuesta a una irritación crónica localizada, una lesión 
traumática o un entorno hormonal alterado. El tratamiento de GP depende de la gravedad de los síntomas (Elias et al.).

Malformaciones vasculares congénitas (MVC). Las malformaciones vasculares congénitas (MCV) son vasos linfáticos o sanguíneos localizados de forma anormal. Por lo general están presentes al nacer, aunque puede no ser aparente (Byung-Boong et al., 2017). Las malformaciones se dividen de acuerdo al tipo de vaso afectado y las características de flujo sanguíneo: flujo lento o rápido, siendo las malformaciones capilares, linfáticas y venosas de flujo lento, mientras que las malformaciones arteriovenosas son de flujo rápido. En la mayoría de los casos, una historia clínica y un examen físico distinguirán una MVC de un hemangioma. La distinción es confirmada por estudios no invasivos. Rara vez se necesita una biopsia de tejido.

Malformaciones capilares (MC). Son anomalías congénitas del desarrollo capilar y suelen aparecer en la dermis superficial. Están presentes al nacer, aumentando gradualmente de tamaño con el crecimiento del niño y generalmente están presentes de por vida sin ninguna tendencia a la involución (Elias et al.). Son malformaciones de flujo lento, con una localización del $70 \%$ en cabeza y cuello. Se extienden intraoralmente y afectan la mucosa, encía y tejidos periodontales (Frigerio \& Tan).

Malformaciones venosas (MV). Las malformaciones venosas son lesiones vasculares benignas que resultan de un desarrollo vascular defectuoso. Estas anomalías no regresan espontáneamente, y son las malformaciones vasculares sintomáticas más comunes detectables en la región de cabeza y cuello. El tipo de lesión suele estar presente al nacer y crece proporcionalmente con la edad. Aproximadamente el $40 \%$ se desarrollan en regiones de cabeza y cuello (Miyazaki et al., 2014; Chen et al., 2015; Wang et al., 2017), suelen ser unilaterales, con un efecto de masa que causa una distorsión significativa y asimetría facial.

Las MV que involucran la lengua, el paladar y la orofaringe a menudo causan deformidades significativas, incluida la maloclusión dental, y representan una condición potencialmente mortal si se introducen en la vía aérea superior junto con lesiones laringofaríngeas (Byung-Boong et al.).

Las malformaciones venosas se dividen en superficiales o profundas, localizadas, multicéntricas o difusas. La piel o mucosa que las cubre varía de color en función de la profundidad y el grado de ectasia de la lesión. Las más superficiales son de color morado, y las profundas más azuladas, verdosas o incluso imperceptibles (Redondo). Son compresibles y sin pulso, aumentan de tamaño y se vuelven sintomáticas con el tiempo. Generalmente son suaves al tacto y se expanden al llenarse de sangre después de una maniobra de Valsalva, o pueden vaciarse cuando se eleva la zona afectada por encima del nivel del corazón.

Las indicaciones para el tratamiento incluyen inflamación recurrente, dolor, deformidad, complicaciones tromboembólicas y sepsis. Las opciones de tratamiento tradicionales son la resección quirúrgica, escleroterapia, terapia con láser o una combinación de éstas (Wang et al.).

Malformaciones linfáticas (ML). Las malformaciones linfáticas son las segundas más comunes después de las malformaciones venosas. Son congénitas, aunque sólo el $65-75 \%$ son diagnosticadas en el nacimiento, alcanzando el $80-90 \%$ al final del segundo año de vida. La localización más frecuente es la cabeza y sobre todo el cuello (90\%). No presentan hiperplasia celular y no son neoplasias, tampoco tienen fases proliferativas e involutivas. Surgen de defectos en la morfogénesis de los vasos linfáticos y consisten en vasos y grandes cámaras revestidas por una sola capa de células endoteliales. Pueden formarse en los primeros años de vida y crecer rápidamente, por lo que requieren tratamiento (Redondo; Elias et al.).

Malformaciones arteriovenosas (MAV). Las malformaciones arteriovenosas son lesiones de alto flujo caracterizadas por la presencia de un nido y una masa central de derivaciones arteriovenosas de baja resistencia que están conectadas a arterias aferentes y venas eferentes. Estas conexiones entre las arterias y las venas son anormales, provocado que la sangre dentro de un órgano o tejido pase por alto la red capilar, lo que ocasiona la pérdida de la regulación sanguínea (Frigerio \& Tan; Elias et al.).

Cerca del $50 \%$ de las lesiones están localizadas en la región oral y maxilofacial, seguido por las extremidades y tronco. La mayoría de las MAV maxilofaciales están localizadas en el centro de la cara, cerca del $70 \%$ involucra la mejilla, nariz, orejas y labio superior. Entre las anormalidades vasculares, las MAV ocurren alrededor de 1,5\%. Aunque son un tipo de enfermedad congénita, sólo el $60 \%$ es descubierto después del nacimiento, el resto es visto en la infancia o adultez (Su et al., 2014). 
Clínicamente se presentan como máculas levemente abultadas, pulsátiles, generalmente con aumento de temperatura de la piel de la región y frémito palpable. Son frecuentes la ulceración, el dolor intenso, el sangrado intermitente y la hipertrofia ósea subyacente a la lesión. MAV que afecta a la piel suelen mostrar una coloración rojiza o azul. En dos tercios de los pacientes afectados se presentan lesiones dentro de la mandíbula. La lesión causa expansión de tabla ósea, movilidad de los dientes circundantes y sangrado gingival asociado (Redondo; Su et al., 2014; Elias et al.; Sierre \& Teplisky, 2016).

El tratamiento endovascular de estas lesiones consiste en la embolización o exclusión del nido malformativo. Se ha postulado que la presencia del nido arteriovenoso es el problema central de la patología, de manera que a menos que la extracción sea completa, la recurrencia es significativa después de la cirugía (Frigerio \& Tan; Sierre \& Teplisky).

En casos de malformaciones grandes e infiltrativas, a menudo se requieren tratamientos multimodales, como cirugía, procedimientos radiológicos de intervención y embolización. La escleroterapia ha sido eficaz, pero la necrosis tisular local y el daño nervioso han sido significativos. La embolización también ha sido una alternativa de tratamiento popular, especialmente cuando se continúa con resección quirúrgica (Frigerio \& Tan).

Uso de polidocanol como tratamiento conservador. Para las malformaciones vasculares especialmente malformaciones linfáticas y venosas, la escisión quirúrgica fue tradicionalmente el tratamiento de elección. Sin embargo, debido a la infiltración y expansión de la lesión en estructuras anatómicas adyacentes, la cirugía no siempre es una opción de tratamiento óptimo y puede conducir a daño de estructuras vecinas a la lesión (Horbach et al., 2016). La escleroterapia convencional con esclerosantes líquidos, es un tratamiento paliativo que ofrece buenos resultados en malformaciones vasculares de tamaño reducido. Está indicada como apoyo preoperatorio de cirugía, buscando la reducción del tamaño de la lesión, como complemento o tratamiento definitivo (Cabrera \& Redondo). La escleroterapia se ha convertido en el método de tratamiento de MV más extendido y apropiado, y se han desarrollado muchos agentes espumantes y líquidos esclerosantes (Górriz-Gómez et al., 2014).

El mecanismo de acción de los agentes esclerosantes es inducir una lesión en la íntima venosa, seguida de una eventual fibrosis del vaso. Los agentes esclerosantes más comúnmente utilizados hoy en día son la solución salina hipertónica, el tetradecil sulfato de sodio y el polidocanol. Las presentaciones más comúnmente ocupadas de estos agentes es de tipo escleroterapia líquida o como espuma. EI polidocanol y tetradecil sulfato son detergentes esclerosantes y se pueden convertir en una espuma mezclándolos con un gas, generalmente aire del medio ambiente, $\mathrm{CO}_{2}, \mathrm{O}_{2}$ (Gibson \& Gunderson, 2018).

El polidocanol es una solución sintética compuesta por hidroxi-polietoxi-dodecano, caña, agua y alcohol etílico al $5 \%$. Originalmente se usaba como anestésico local, pero en la actualidad se usa casi exclusivamente como un esclerosante (Górriz-Gómez et al.). Es un detergente no iónico de uso común que genera lisis del revestimiento endotelial a través de la absorción en la membrana celular y desnaturalización de proteínas por sobrehidratación rápida de las células endoteliales, generando una lesión vascular. Estimula la agregación plaquetaria para posteriormente formar una densa red de plaquetas y fibrina, y eventualmente la fibrosis del vaso sanguíneo (Mimura et al., 2009; Horbach et al.; Gibson \& Gunderson).

Este agente esclerosante es seguro, presenta una tasa mucho menor de efectos no deseados, las alergias son bajas, la inyección es indolora y la necrosis de la piel es poco frecuente. El mayor inconveniente es su capacidad para pigmentar las venas (GórrizGómez et al.).

Nuestro primer caso presentado corresponde a una malformación arteriovenosa, su diagnóstico fue conducido por la exploración clínica y la ecografía Doppler, posteriormente confirmado con el estudio histopatológico. En primera instancia fue tratada de forma conservadora mediante infiltraciones intralesionales de polidocanol $1 \%$ (agente esclerosante). Si bien disminuyó en tamaño, no se logró la remisión completa de la lesión, esto según Jafarian et al. (2016) se debe a que las MAV tienen estados hemodinámicos de alto flujo que pueden lavar los agentes esclerosantes, impidiendo el resultado terapéutico esperado. Por otra parte, la malformación arteriovenosa de este caso, al estar ubicada en una región altamente irrigada, recibe alimentación de diferentes vasos que pueden hacer de colaterales a los vasos esclerosados y mantener la irrigación de la lesión. En vista a estos resultados, se optó por realizar una escisión quirúrgica completa de la lesión, considerando la previa embolización de los vasos san- 
guíneos, la ligadura y cauterización de éstos para evitar hemorragias. Se realiza la resección completa de la lesión ya que es el método quirúrgico más efectivo para las MAV, descartando otros métodos quirúrgicos menos agresivos descritos como el curetaje y la resección parcial que causan el restablecimiento de la lesión y también conducen a una recurrencia rápida, con el riesgo de estar acompañados por un sangrado intraoperatorio.

El segundo caso reportado presenta un diagnóstico de malformación venosa. El diagnóstico fue determinado principalmente por la clínica del paciente. El tratamiento con infiltraciones de polidocanol al 2 $\%$ en dos sesiones, dio resultados favorables. Es así que la escleroterapia representa una alternativa valiosa para tratar este tipo de malformación vascular como se ha mencionado anteriormente. De hecho, además ofrece la ventaja de no dejar cicatrices externas, como se evidencia en nuestro caso.

El uso de agentes esclerosantes es uno de los tratamientos conservadores de primera elección al momento de tratar malformaciones vasculares, junto a otras opciones terapéuticas como láser y la embolización. No obstante, si el tratamiento no erradica por completo la lesión, se opta por un tratamiento quirúrgico, siendo la escleroterapia un complemento a la cirugía, como se evidencia en el primer caso clínico presentado. Actualmente un enfoque terapéutico integrado como lo es el uso combinado de la escleroterapia - la embolización con la cirugía, obtiene mejores resultados, de manera de reducir el riesgo y las complicaciones que existen cuando la cirugía se realiza de forma aislada (Fernández Alonso, 2004). Por lo tanto, el tratamiento quirúrgico para este tipo de lesiones debe considerarse, no como una herramienta de tratamiento única sino como una terapia asociada a otras opciones conservadoras, con el objetivo de otorgar un mejor resultado terapéutico con mínimas comorbilidades.

\section{CONCLUSIONES}

Las anomalías vasculares comprenden dos grupos: tumores vasculares y malformaciones vasculares que a menudo requieren un tratamiento multidisciplinario. El diagnóstico es determinado por la clínica e imágenes. Los métodos por imágenes son un pilar fundamental para el adecuado diagnóstico de estas patologías y su posterior clasificación, sobre la cual se basará el tratamiento específico. Los tratamientos son amplios, desde conservadores hasta quirúrgicos, existiendo un amplio abanico de opciones terapéuticas seguras y efectivas para el manejo de estas anomalías. El tratamiento esclerosante es un tratamiento conservador que tiene por objetivo causar una lesión de las paredes del endotelio de los vasos sanguíneos, disminuyendo así el flujo sanguíneo y posteriormente su fibrosis.

Para este tipo de lesiones es necesario evaluar la condición clínica tanto del paciente como de la lesión propiamente tal, para así plantear un tratamiento adecuado y acorde a las necesidades clínicas del caso.

TORRES, M. C.; SANTANA, L. J.; BRAVO, A. R. \& MARDONES, M. M. Vascular anomalies of the oral cavity: Review of classification and treatment applied to two clinical cases. Int. J. Odontostomat., 14(1):48-54, 2020.

ABSTRACT: Vascular anomalies of the head and neck are a group of lesions that affect blood and lymph vessels where treatment remains a challenge. The updated classification of head and neck vascular anomalies is the modified Mulliken classification, which subdivides them into a) vascular tumors and b) vascular malformations. In this report, we present two clinical cases of male patients, with diagnosis of vascular anomalies affecting the lip and hard palate, diagnosed as arteriovenous malformation and venous malformation, respectively. These lesions were completely treated with conservative (sclerosing agents) and/or surgical (complete surgical exeresis of the lesion) treatments, achieving a complete remission. Consequently, we present a review of the literature focused on the current classification, current and future therapeutic approaches.

KEY WORDS: vascular anomalies, vascular malformation, vascular neoplasms, oral cavity.

\section{REFERENCIAS BIBLIOGRÁFICAS}

Behnia, H.; Jafarian, M.; Dehghani, N.; Dehghani, S. \& Seyedan, K. Comprehensive treatment and rehabilitation of a patient with maxillary arteriovenous malformation. J. Craniofac. Surg., 25(5):e463-7, 2014.

Byung-Boong, L.; Laredo, J. \& Neville, R. Congenital Vascular Malformations: General Considerations. En: Kim, Y. B.; Lee, B. B.; Yakes, W. F. \& Do, Y. S. (Eds.). Congenital Vascular Malformations. A Comprehensive Review of Current Management. Berlin, Springer Nature, 2017. pp.41-5.

Cabrera, J. \& Redondo, P. Sclerosing treatment of vascular malformations. An. Sist. Sanit. Navar., 27 Suppl. 1:117-26, 2004.

Chen, A. W.; Liu, Y. R.; Li, K.; Zhang, K.; Wang, T. \& Liu, S. H. Efficacy of sclerotherapy with radio-opaque foam guided by digital 
subtraction angiography for the treatment of complex venous malformations of the head and neck. Br. J. Oral Maxillofac. Surg., 53(9):809-13, 2015.

da Silva, W. B.; Ribeiro, A. L.; de Menezes, S. A.; de Jesus Viana Pinheiro, J. \& de Melo Alves-Junior, S. Oral capillary hemangioma: a clinical protocol of diagnosis and treatment in adults. Oral Maxillofac. Surg., 18(4):431-7, 2014.

Elias, G.; McMillan, K. \& Monaghan, A. Vascular Lesions of the Head and Oral Cavity - Diagnosis and Management. Dent. Update, 43(9):859-60, 862-4, 866, 2016.

Fernández Alonso, L. Surgical treatment of vascular malformations. An. Sist. Sanit. Navar., 27 Suppl. 1:127-32, 2004.

Frigerio, A. \& Tan, O. T. Laser applications for benign oral lesions. Lasers Surg. Med., 47(8):643-50, 2015.

Gibson, K. \& Gunderson, K. Liquid and foam sclerotherapy for spider and varicose veins. Surg. Clin. North Am., 98(2):415-29, 2018.

Górriz-Gómez, E.; Vicente-Barrero, M.; Loras-Caballero, M. L.; Bocanegra-Pérez, S.; Castellano-Navarro, J. M.; Pérez-Plasencia, D. \& Ramos-Macías, A. Sclerotherapy of face and oral cavity low flow vascular malformations: our experience. Br. J. Oral Maxillofac. Surg., 52(1):43-7, 2014.

Gupta, A.; Verma, A.; Dhua, A. \& Bhatnagar, V. Vascular Anomalies: A Pediatric Surgeon's Perspective. Indian J. Pediatr., 84(8):6127, 2017.

Horbach, S. E.; Lokhorst, M. M.; Saeed, P.; de Goüyon Matignon de Pontouraude, C. M.; Rothová, A. \& van der Horst, C. M. Sclerotherapy for low-flow vascular malformations of the head and neck: A systematic review of sclerosing agents. J. Plast. Reconstr. Aesthet. Surg., 69(3):295-304, 2016.

Jafarian, M.; Dehghani, N.; Shams, S.; Esmaeelinejad, M. \& Aghdashi, F. Comprehensive treatment of upper lip arteriovenous malformation. J. Maxillofac. Oral Surg., 15(3):394-9, 2016.

Kim, Y. B.; Lee, B. B.; Yakes, W. F. \& Do, Y. S. (Eds.). Congenital Vascular Malformations. A Comprehensive Review of Current Management. Berlin, Springer Nature, 2017.

Léauté-Labrèze, C.; Dumas de la Roque, E.; Hubiche, T.; Boralevi, F.; Thambo, J. B. \& Taïeb, A. Propranolol for severe hemangiomas of infancy. N. Engl. J. Med., 358(24):2649-51, 2008.

Medeiros, R. Jr.; Silva, I. H.; Carvalho, A. T.; Leão, J. C. \& Gueiros, L. A. Nd:YAG laser photocoagulation of benign oral vascular lesions: a case series. Lasers Med. Sci., 30(8):2215-20, 2015.

Mimura, H.; Fujiwara, H.; Hiraki, T.; Gobara, H.; Mukai, T.; Hyodo, T.; Iguchi, T.; Yasui, K.; Kimata, Y. \& Kanazawa, S. Polidocanol sclerotherapy for painful venous malformations: evaluation of safety and efficacy in pain relief. Eur. Radiol., 19(10):2474-80, 2009.

Miyazaki, H.; Romeo, U.; Ohshiro, T.; Kudo, T.; Makiguchi, T.; Kawachi, N.; Ogawa, M.; Inoue, Y. \& Yokoo, S. Treatment strategies for large oral venous malformations using intralesional laser photocoagulation. Lasers Med Sci., 29(6):1987-90, 2014.

Mulligan, P. R.; Prajapati, H. J.; Martin, L. G. \& Patel, T. H. Vascular anomalies: classification, imaging characteristics and implications for interventional radiology treatment approaches. Br. J. Radiol., 87(1035):20130392, 2014.

Mulliken, J. B. \& Glowacki, J. Hemangiomas and vascular malformations in infants and children: a classification based on endothelial characteristics. Plast. Reconstr. Surg., 69(3):412-22, 1982.

Redondo, P. Classification of vascular anomalies (tumours and malformations). Clinical characteristics and natural history. An. Sist. Sanit. Navar., 27 Suppl. 1:9-25, 2004.

Sierre, S. \& Teplisky, D. Diagnóstico y tratamiento de las malformaciones vasculares en niños. Estado actual y estado del arte. Rev. Interv., 16(3):30-48, 2016.

Su, L. X.; Fan, X. D.; Zheng, J. W.; Wang, Y. A.; Qin, Z. P.; Wang, X. K.; Yang, Y. W. \& Zhao, Y. F. A practical guide for diagnosis and treatment of arteriovenous malformations in the oral and maxillofacial region. Chin. J. Dent. Res., 17(2):85-9, 2014.
Wang, D.; Su, L.; Han, Y.; Wang, Z.; Zheng, L.; Li, J. \& Fan, X. Direct intralesional ethanol sclerotherapy of extensive venous malformations with oropharyngeal involvement after a temporary tracheotomy in the head and neck: Initial results. Head Neck, 39(2):288-96, 2017.

Direccion para correspondencia:

Coral Torres M.

Servicio de Cirugía

Hospital San José

Santiago

CHILE

Email: torresm.coral@gmail.com

Recibido: 29-07-2019

Aceptado: 19-08-2019 